\title{
OH/IR Star Color Mimics with S(25) > 100 Jy
}

\author{
B. M. Lewis
}

Arecibo Observatory, Puerto Rico

O-rich circumstellar dust shells that do not exhibit $1612 \mathrm{MHz}$ masers (Lewis 1992), despite appropriate IR colors and $1612 \mathrm{MHz}$ sensitivity, are known as OH/IR star color mimics. Since previous studies of mimics depend on samples with a limited IR spectral type and color range, we use the data collected for poster III-8 to identify the mimics in an all-sky sample of bright IRAS sources with $\mathrm{S}(25)>100 \mathrm{Jy}$ and $(60-25) \mu \mathrm{m}<0.0$ : this expands on Gaylard et al. (1989), and benefits from the greater quantity of maser data now and a wider IR color range.

It is advantageous to use the $\mathrm{S}(25)>100 \mathrm{Jy}$ sources since their IR colors are as trustworthy as can presently be specified, and many of them have been examined at a variety of wavelengths. In poster III- 8 we find (i) that the red limit to the predictable color range of OH/IR stars with $\mathrm{S}(25)>100 \mathrm{Jy}$ is well determined at $(25-12) \mu \mathrm{m}<+0.1$; (ii) that the carbon or O-rich flavor of most shells can be determined from the LRS spectrum and/or complement of masers. And $1612 \mathrm{MHz}$ observations are extant for all but 2 of the potential OH/IR stars with (25-12) $\mu \mathrm{m}>-0.5$. Consequently most emission-line objects and pre main sequence (PMS) stars can be identified. It is also clear that while $1612 \mathrm{MHz}$ detections are rare when (25-12) $\mu \mathrm{m}<-0.55$, most of the blue objects exhibit other masers. We therefore limit our working definition of "mimics" to O-rich shells without $1612 \mathrm{MHz}$ masers having $+0.1>(25-12) \mu \mathrm{m}>-0.5$, that are not PMS, emission-line or carbon stars.

This study identifies 17 mimics, of which two are presumptively proto planetary nebulae (PPN) with "odd" colors. Five of these exhibit mainline $\mathrm{OH}$ masers and three exhibit water masers, though $<50 \%$ have been searched for either. Most mimics lie near the blue bound on their (25-12) $\mu \mathrm{m}$ color range, as did the LRS $2 \mathrm{n}-3 \mathrm{n}$ mimics in the Arecibo sky. There is no statistically discernible difference in the color distribution of O-rich mimics and $\mathrm{OH} / \mathrm{IR}$ stars in color-color plots, except that there are very few red mimics. Since the ratio of $1612 \mathrm{MHz}$ intensity to $\mathrm{S}(25)$ decreases in OH/IR stars as (25-12) $\mu \mathrm{m}$ gets bluer, the difference in this ratio between the mimics and OH/IR stars decreases markedly when estimated from extant, survey-quality $1612 \mathrm{MHz}$ data. This suggests a need for more sensitive $1612 \mathrm{MHz}$ observations of mimics to confirm the absence of emission to a more stringent level. Nevertheless so many mimics are already detected with mainline $\mathrm{OH}$ emission that they are unlikely to be simply normal OH/IR stars with weaker than usual $1612 \mathrm{MHz}$ emission. The reddest IRAS sources with weak $1612 \mathrm{MHz}$ masers, and hence small values of $I_{1612} / \mathrm{S}(25)$, are often PPN or PMS regions: neither attribution can explain the existence of the bluer mimics.

\section{REFERENCES}

Gaylard, M. J., et al., 1989, MNRAS, 236, 247.

Lewis, B. M., 1992, Ap. J., 396, 251. 